\title{
Cancer du col de l'utérus : classification et explorations préthérapeutiques
}

C De Ollveira

F Mota

\begin{abstract}
Résumé. - Cet article décrit dans ses grandes lignes la classification du cancer du col utérin. II met l'accent sur l'importance d'un examen clinique pratiqué sous anesthésie et analyse les explorations complémentaires et examens annexes acceptés pour le bilan d'extension du cancer. Les techniques optionnelles susceptibles d'améliorer la classification clinique sont brièvement présentées. Les auteurs soulignent que déterminer le stade d'un cancer du col ne limite en rien les stratégies thérápeutiques disponibles, qui doivent être adaptées à la maladie sur une base individuelle.
\end{abstract}

- 2002 Editions Scientifiques et Médicales Elsevier SAS. Tous droits réservis.

Mots-clés : cystoscopie, urographie intraveineuse, lymphographie, rectosigmoïdoscopie.

\section{Introduction}

Une classification (bilan d'extension de la maladie) précise du cancer du col est nécessaire à la sélection des modalités thérapeutiques appropriées et à la planification de leur séquence. Cette caractérisation eśs importante, non seulement car elle permet d'évaluer les stratégies thérapeutiques au sein d'un même établissement, mais aussi car elle offre un moyen de comparer les résultats obtenus par différents établissements à travers le monde. Par conséquent, elle doit être aussi uniforme que possible. Une classification précise est également essentielle à l'optimisation des résultats du traitement, car la prise en charge des patientes et le pronostic varient considérablement selon le stade de la maladie. Toutefois, la classification ne limite pas les modalités de traitement disponibles et les soins entrepris peuvent être personnalisés en fonction de l'architecture tumorale spécifique de chaque patiente.

\section{Classification clinique}

La classification du cancer du col est reconnue comme étant un processus essentiellement clinique, qui doit de préférence être pratiqué sous anesthésie. Il est impossible de revenir par la suite sur le bilan établi, au cas où une intervention ou un traitement ultérieurs révèleraient un stade plus avancé de la maladie, car ce " passage au stade supérieur $\bowtie$ conduirait à de fausses améliorations dans les résultats du traitement $d^{\prime} u n$ stade précoce. Les explorations préthérapeutiques pouvant être employées pour la caractérisation du cancer du col utérin sont présentées dans le tableau I.

\section{EXAMEN CLINIQUE}

\section{ET EXPLORATIONS COMPLEMENTAIRES}

La grande majorité des patientes présente un examen clinique général normal. Toutefois, les ganglions lymphatiques inguinaux

\footnotetext{
Carlos De Ollvetra : Professeur de gymécologie, chet du service d'ancologie gnmécalogique. Fernando Mota : Professeur auxilioire de gmécologie.

Service de gnécologie, hópitol universitaire, 3049 Coimbra, Partugat.
}

\begin{tabular}{l|l}
\hline \multicolumn{2}{l}{ Tablean 1. - Explorations préthérapeutiques. } \\
\hline Examen clinique & $\begin{array}{l}\text { Examen physique général } \\
\text { Examen génital } \\
\text { Examen rectovaginal bimanuel (sous anesthésie) }\end{array}$ \\
\hline Interventions & $\begin{array}{l}\text { Colposcopie } \\
\text { Biopsie } \\
\text { Curetage endocervical } \\
\text { Conisation }\end{array}$ \\
\hline Examens annexes & $\begin{array}{l}\text { Urographie intraveineuse } \\
\text { Cystoscopie } \\
\text { Rectosigmoïdoscopie } \\
\text { Radiographie thoracique } \\
\text { Radiographie osseuse }\end{array}$ \\
\hline Examens optionnels & $\begin{array}{l}\text { Lymphographie } \\
\text { Tomodensitométrie } \\
\text { Echographie } \\
\text { Imagerie par résonarce magnétique } \\
\text { Scintigraphie } \\
\text { Laparoscopie }\end{array}$ \\
\hline
\end{tabular}

doivent être palpés, en particulier en cas d'invasion du tiers inférieur du vagin. Les ganglions lymphatiques supraclaviculaires doivent également être palpés, car ils peuvent être le site de métastases à distance, même dans les cancers du col à un stade apparemment précoce. Une ascite ou une hépatomégalie sont recherchées par palpation abdominale. L'étiologie d'un épanchement pleural ou d'un gonflement des jambes doit être recherchée. Tous ces signes peuvent indiquer la présence de métastases.

L'examen de la vulve et du périnée peut, dans de rares cas, révéler une lésion in situ ou cancéreuse. Le lien entre le méat urétral et une lésion éventuelle dans le tiers inférieur du vagin est enregistré. Toutes les lésions suspectes doivent faire l'objet $d^{\prime}$ une biopsie, afin de confirmer le diagnostic de métastases.

Lors de l'examen au spéculum, le col peut apparaître parfaitement normal si le cancer est très peu étendu (subclinique) ou situé au niveau de l'endocol. Chez les patientes dont l'examen cytologique des frottis vaginaux laisse suspecter la présence d'un cancer invasif précoce, mais dont le col a une apparence normale, une colposcopie est obligatoire et identifie la zone la plus suspecte devant être biopsiée. Les observations colposcopiques suggérant un cancer 
précoce du col sont les suivantes : vaisseaux sanguins atypiques (anormaux en taille, forme, calibre, direction), contours de surface irréguliers, épithélium ulcéré, friable et jaune orangé, présence d'anomalies colposcopiques étendues et sévères ou complexes et leur extension dans le canal.

L'incidence des adénocarcinomes du col augmente; ils représentent environ $20 \%$ des cancers cervicaux. L'adénocarcinome a parfois l'apparence d'une lésion papillaire dans la portion sus-vaginale du col. Mais il se développe le plus souvent à l'intérieur du canal alors que l'exocol apparaît parfaitement normal, au moins dans un premier temps. Dans ces circonstances, un curetage endocervical doit obligatoirement constituer la dernière étape d'un examen colposcopique soigneux.

Les biopsies à l'emporte-pièce sont appropriées pour confirmer un cancer cliniquement évident. Toutefois, si la biopsie ou le curetage endocervical ne permet pas d'établir le diagnostic avec certitude, une conisation diagnostique est nécessaire. En outre, les biopsies à l'emporte-pièce ne suffisent pas au diagnostic définitif du cancer micro-invasif du col. Dans ce cas également, une conisation est indiquée pour évaluer correctement la profondeur et l'étendue horizontale de la micro-invasion, ainsi que la pénétration vasculaire. La lésion de l'exocol peut être exophytique, avec un aspect en " chou-fleur ", irrégulier, hémorragique, de taille variable, parfois avec une marge élevée et ferme. La lésion peut également être ulcérée avec une base indurée, auquel cas le col et éventuellement les culs-de-sac vaginaux peuvent être remplacés par un cratère nécrotique. Parfois, une tumeur infiltrante présente une petite ulcération visible ou une masse exophytique, mais le col est alors perçu à la palpation comme étant dur comme de la pierre. Avec l'extension de la tumeur, le col peut apparaître anormalement gros et lourd (col en « tonneau n) lorsqu'il est entièrement envahi par un carcinome épidermoïde ou lorsqu'une tumeur endocervicale infiltrante se dévelọppe à l'intérieur du canal. Après l'examen du col, les parois vaginales et en particulier les culs-de-sac vaginaux sont inspectés avec soin afin de rechercher d'éventuelles lésions suspectes. Des biopsies doivent être pratiquées.

\section{EXPLORATION PELVIENNE SOUS ANESTHESIE}

Cette étape de l'évaluation, réalisée par au moins un, généralement deux, cancérologues expérimentés, est essentielle à la caractérisation du cancer du col utérin car, en l'absence d'exploration rectovaginale sous anesthésie, la véritable étendue du cancer peut être sousestimée. Cet examen est indispensable à l'évaluation de l'extension de la tumeur en direction des culs-de-sac vaginaux, vers le bas dans le vagin, latéralement au niveau des paramètres, antérieurement dans l'espace vésicovaginal ou postérieurement dans les ligaments utérosacrés. La palpation vaginale permet de déterminer le volume tumoral, ainsi que la taille et la consistance du col. Un col utérin extrêmement dur à la palpation peut être révélateur d'un cancer endophytique ou infiltrant. Les lésions vaginales infiltrantes peuvent également être décelées de cette manière, mais passent souvent inaperçues lors de l'inspection vaginale.

Une palpation transrectale sous anesthésie est indispensable lors de l'évaluation paramétriale.

- Les paramètres sont-ils souples/élastiques ou nodulaires et envahis par la tumeur? La tumeur est souvent infectée secondairement. Pour cette raison, il est parfois impossible de savoir dans quelle mesure la fixation et l'épaississement des paramètres sont dus à la tumeur ou à une réaction inflammatoire. Un traitement antibiotique et anti-inflammatoire préliminaire peut être prescrit.

- L'invasion est-elle unilatérale ou bilatérale ?

- Y a-t-il du tissu fixé sur la paroi pelvienne? Il arrive que l'on puisse sentir un ligament utérosacré nodulaire/envahi. Un élargissement ganglionnaire pelvien peut être décelé à la palpation: Le gynécologue évalue la souplesse et la mobilité ou l'invasion du rectum. L'envahissement éventuel de l'espace rectovaginal peut également être détectê en introduisant l'index à l'intérieur du vagin et le majeur à l'intérieur du rectum.

\section{Examens de laboratoire}

Une évaluation biologique générale, impliquant des examens cliniques, mais également toute une série de tests de laboratoire, permet d'évaluer l'état de santé généra] de la patiente, ainsi que tout phénomène métabolique, rénal ou cardiorespiratoire susceptible de contre-indiquer une intervention chirurgicale. En outre, le taux d'hémoglobine doit être déterminé et l'anémie corrigée. Des tests fonctionnels hépatiques anormaux peuvent indiquer la présence de métastases dans le foie. Une hypercalcémie peut être un signe de maladie avancée avec atteinte osseuse.

La recherche du marqueur biologique squamous cell carcinoma (SCC) pour le carcinome épidermoïde est nécessaire avant l'instauration de tout traitement. Si le taux est élevé, le marqueur peut s'avérer utile pour l'évaluation de la réponse au traitement. Un taux sérique élevé est observé dans 50 à $75 \%$ des cancers localement évolués et le titre est corrélé au stade, au volume tumoral et au pronostic. Dans les adénocarcinomes du col, une mesure du marqueur tumoral CA125 doit être obtenue avant l'intervention.

\section{Examens annexes pour la classification}

Les techniques utilisées pour établir le bilan d'extension du cancer du col utérin et les examens annexes possibles permettant d'améliorer l'évaluation clinique sont répertoriés dans le tableau I. Ce tableau mentionne également un certain nombre d'examens optionnels, dont les résultats $n$ 'ont pas d'incidence sur la classification FIGO (Fédération internationale de gynécologie et d'obstétrique). Ces examens ne sont pas employés pour la détermination du stade FIGO, car ils ne sont pas disponibles partout dans le monde, et leur interprétation est variable. Toutefois, les informations fournies par ces approches facultatives peuvent être utilisées pour planifier le traitement.

\section{UROGRAPHIE INTRAVEINEUSE}

Cet examen est souvent normal. Toutefois, il met parfois en évidence des uretères doubles, dont il est essentiel de connaitre la position avant d'entreprendre un acte chirurgical. La détection d'un rein pelvien doit être prise en compte lors de la délimitation des champs d'irradiation pelvienne pour la radiothérapie. Des anomalies urétérales, vésicales ou rénales peuvent parfois être observées, en particulier dans les cancers évolués du col. Toute déviation, angulation, rigidité ou obstruction des uretères doit être enregistrée, car ils peuvent être directement envahis par l'extension régionale de la tumeur (en particulier dans le voisinage de la vessie) ou être comprimés ou déviés par une adénopathie.

Une hydronéphrose, un retard d'excrétion ou un dysfonctionnement rénal peuvent être observés. La vessie peut présenter un empiètement évoquant une compression, ou encore une irrégularité et/ou une rigidité suggérant un envahissemeñt par la tumeur.

\section{CYSTOSCOPIE}

II est rare que la cystoscopie apporte des informations intéressantes dans l'évaluation des cancers du col de stades I et II. Toutefois, cet examen peut aider à établìr l'intégrité ou l'invasion de la vessie. Une inspection attentive de la muqueuse vésicale, ainsi que du trigone et de l'orifice des uretères, doit être entreprise. Une muqueuse vésicale rose normale peut être le site d'un érythème ou d'une leucoplasie. Les croissances exophytiques uniques ou multiples, granulations, ulcérations et œdèmes localisés sont autant de signes d'invasion de la muqueuse vésicale. Des biopsies de ces zones suspectes sont nécessaires pour confirmer le diagnostic. II est toutefois important de rappeler qu'un envahissement sous-muqueux de la vessie n'est pas toujours visible à la cystoscopie.

\section{RECTOSIGMOIDOSCOPIE}

Ce type d'examen n'est utile qu'en cas d'invasion tumorale postérieure et d'infiltration de l'espace rectovaginal. Il permet d'observer la muqueuse du rectum et du côlon inférieur, qui peut 
être normale ou congestive, fixe, présenter des végétations friables et sanguinolentes. La lumière peut également être sténosée par une extension de la tumeur cervicale jusqu'à la couche musculaire. Des biopsies doivent être pratiquées.

\section{RADIOGRAPHIE THORACIQUE (FACE ET PROFIL)}

Bien que les métaștases pulmonaires soient rares, leur présence éventuelle doit être écartée. Des métastases pulmonaires existent dans environ $5 \%$ des cas de maladies évoluées qui, en leur absence, seraient considérées comme des stades III ou IVa. La radiographie thoracique permet par ailleurs à l'anesthésiste d'évaluer le statut cardiorespiratoire de la patiente.

\section{RADIOGRAPHIE OSSEUSE}

Cet examen présente peu d'intérêt, car les métastases osseuseş sont rares et souvent symptomatiques.

\section{Système de classification de la FIGO}

Le système actuel de classification du cancer du col utérin établi par la FIGO est présenté dans le tableau II ${ }^{151}$. Cette classification ne s'appliquant qu'aux carcinomes, une confirmation histologique de la maladie est nécessaire. En cas de doute lors de la caractérisation du stade de la maladie, le stade retenu doit être le plus précoce. Le diagnostic des stades Ia1 et Ia2 doit reposer sur l'examen microscopique du tissu prélevé, de préférence un cône incluant la totalité de la lésion. La profondeur d'invasion ne doit pas dépasser $5 \mathrm{~mm}$ entre la base de l'épithélium de surface ou glandulaire, où elle prend son origine, et le point d'envahissement le plus profond. La seconde dimension, l'étendue horizontale, ne doit pas excéder $7 \mathrm{~mm}$. L'infiltration de l'espace vasculaire veineux ou lymphatique n'a aucune incidence sur la classification, mais elle doit être enregistrée car elle peut influencer les décisions thérapeutiques

Tableau II. - Classification de la Fédération internationale de gynécologie et obstétrique des cancers du col utérin.

\begin{tabular}{|c|c|}
\hline \multicolumn{2}{|r|}{ Cancer preinvasif } \\
\hline Stade 0 & $\begin{array}{l}\text { Carcinome in situ, carcinome intraépithélial (ces cas ne doivent pas } \\
\text { être inclus dans les statistiques thérapentiques) }\end{array}$ \\
\hline \multicolumn{2}{|r|}{ Cancer invasif } \\
\hline Stade I & $\begin{array}{l}\text { Cancer strictement limité au col utérin ('́extension au corps utérin } \\
\text { ne doit pas être prise en compte) }\end{array}$ \\
\hline Stade Ia & $\begin{array}{l}\text { Cancer préclinique, c'est-à-dire diagnostiqué uniquement par } \\
\text { microscopie }\end{array}$ \\
\hline Stade lal & Lésiors avec invasion $\leq 3 \mathrm{~mm}$ \\
\hline Stade la2 & $\begin{array}{l}\text { Lésions détectées au microscope pouvant être mesurées. Profon- } \\
\text { deur d'invasion > }>3 \text { a } 5 \mathrm{~mm} \text {. L'extension horizontale ne doit pas } \\
\text { dépasser } 7 \mathrm{~mm}\end{array}$ \\
\hline Stade $\mathbf{b}$ & Lésions invasives > $5 \mathrm{~mm}$ \\
\hline Stade Ibl & Lésions $\leq 4 \mathrm{~cm}$ \\
\hline Stade Ib2 & Lésions $>4 \mathrm{~cm}$ \\
\hline Stade 11 & Cancer étendu au-delà du col, sans atteindre la paroi pelvienne \\
\hline $\begin{array}{l}\text { Stade IIa } \\
\text { Stade IIb }\end{array}$ & $\begin{array}{l}\text { Cancer infiltrant le vagin, mais pas le tiers inférieur } \\
\text { Pas d'infiltration visible des paramètres } \\
\text { Infiltration visible des paramètres }\end{array}$ \\
\hline Stade III & $\begin{array}{l}\text { Cancer étendu à la paroi pelvienne. L'examen rectal ne fait pas } \\
\text { apparaitre d'espace non cancéreux entre la tumeur et la paroi } \\
\text { pelvienne. La tumeur atteint le tiers inférieur du vagin. Tous les } \\
\text { cas avec hydronéphrose ou dysfonctionnement rénal (sauf en pré- } \\
\text { sence d'une autre cause connue) }\end{array}$ \\
\hline Stade Illa & Pas d'extension a la paroi pelvienne \\
\hline Stade IIIb & $\begin{array}{l}\text { Extension à la paroi pelvienne et/ou hydronéphrose ou dysfonc-, } \\
\text { tionnement rénal }\end{array}$ \\
\hline Stade IV & $\begin{array}{l}\text { Cancer étendu au-delà du pelvis nél ou envahissant cliniquement } \\
\text { la muqueuse de la vessie ou du rectum }\end{array}$ \\
\hline Stade IVa & Extension de la tumeur aux organes voisins \\
\hline Stade IVb & Extersion à des organes éloignés \\
\hline
\end{tabular}

futures. Les lésions plus étendues doivent être classées comme des stades $\mathrm{lb}$. En règle générale, il est impossible de déterminer cliniquement si un cancer du col a atteint le corps utérin. Il ne doi donc pas être tenu compte de l'extension au corps utérin.

Certains auteurs sont favorables à la sous-division du stade la en "invasion stromale précoce" (bourgeons néoplasiques épithéliaux microscopiques émanant de la base d'un cancer in situ) et " microinvasion » jusqu'à une profondeur comprise entre 1 et $5 \mathrm{~mm}$ affirmant que les deux entités histopathologiques diffèren significativement en termes de récidive, d'invasion vasculaire et de survie ${ }^{[3]}$. L'objet de cette classification est d'identifier un groupe de patientes ne présentant pas de risque de métastases lymphatiques ou de récidive, et pouvant être traitées de manière conservatrice.

Une patiente présentant une tumeur fixée à la paroi pelvienne par un paramètre court et induré, mais non nodulaire, appartient au stade IIb. A I'examen clinique, il est impossible de déterminer si un paramètre lisse et induré est réellement cancéreux ou seulement inflammatoire. Par conséquent, la maladie n'est considérée comme de stade III que si le paramètre est nodulaire sur la paroi pelvienne ou si la tumeur elle-mème s'étend à la paroi pelvienne. La présence d'une hydronéphrose ou d'un dysfonctionnement rénal dû à la sténose de l'uretère par le cancer entraîne une classification comme stade III, même si les autres paramètres conduiraient à un diagnostic de stade I ou II.

La présence d'un œdème bulleux ne permet pas, à elle seule, d'établir un stade IV. Des crêtes et sillons dans la paroi vésicale doivent être interprétés comme des signes d'infiltration vésicale souis-muqueuse s'ils restent fixés à la tumeur à la palpation (examen depuis le vagin ou le rectum durant la cystoscopie). Enfin, l'observation cytologique de cellules malignes dans les lavages de la vessie nécessite des examens complémentaires, et un échantillon de biopsie doit être prélevé sur la muqueuse vésicale.

\section{Examens optionnels}

Il est évident qu'aucune des approches et techniques décrites précédemment ne fournit d'informations sur la voie métastatique la plus courante du cancer du col : le système lymphatique. Il est utile de connaître le statut des ganglions lymphatiques, entre autres, afin d'établir un programme thérapeutique adapté à la patiente. Les approches employées à cet effet sont la lymphographie, l'échographie et, plus récemment, la tomodensitométrie (scanner), l'ìmagerie par résonance magnétique (IRM) et même la biopsie par aspiration des ganglions lymphatiques à l'aiguille fine.

Il convient de souligner que les résultats de ces examens n'ont aucune incidence sur le stade rapporté pour une patiente, mais qu'ils peuvent influer sur son traitement, en permettant d'individualiser le programme thérapeutique en fonction du stade et de la maladie. En outre, les résultats des techniques d'imagerie font apparaitre les limites de la caractérisation clinique.

\section{LYMPHOGRAPHIE}

La lymphographie étant le seul examen portant sur l'architecture interne des ganglions lymphatiques, elle est, au moins théoriquement, très importante. Pourtant, cette technique ne fait pas l'unanimité. II s'agit d'une -étude assez difficile, peu sensible mais très spécifique, et certains cancérologues estiment que son rôle dans la prise en charge future des patientes atteintes d'un cancer du col est contestable. La sensibilité la plus faible est observée avec les métastases de petite taille et même les dépôts ganglionnaires de grande taille peuvent ne pas être décelés.

Une image radiotransparente à l'intérieur d'un ganglion élargi est particulièrement suggestive $d^{\prime}$ un envahissement ganglionnaire, de même qu'une obstruction ou une asymétrie des ganglions lymphatiques pelviens et para-aortiques ${ }^{(111}$. Piver et al ${ }^{[12]}$ estiment que la lymphographie permet de détecter $78 \%$ des envahissements lymphatiques mis en évidence par les méthodes histopathologiques. 
Fuchs et Rosenberg ${ }^{[7]}$ ont montré un pourcentage de diagnostics exacts de $87 \%$, avec $1,5 \%$ de résultats faussement positifs et $12 \%$ de résultats faussement négatifs. Les principaux facteurs contribuant a ces taux sont les suivants: absence congénitale de certains ganglions lymphatiques, non-opacification de certains ganglions pelviens et para-aortiques, inflammation et taille des métastases (elles doiveñt mesurer de $5 \mathrm{a} 10 \mathrm{~mm}$ pour être visibles ${ }^{[6]}$ ). Une tuberculose ou une endométriose peuvent également conduire à des résultats faussement positifs. La lymphographie possède toutefois certains avantages. Sa valeur pronostique a été démontrée dans les cancers de stade III. Le taux global de survie à 5 ans est de $58 \%$ contre $17 \%$, si l'on compare les résultats négatifs et positifs de lymphographie ${ }^{[7]}$. Hammond et al ${ }^{[8]}$ sont parvenus à des conclusions similaires pour les cancers du col de stades Ib à IIIb. En outre, il est possible de prélever les ganglions para-aortiques suspects durant la chirurgie ou d'étendre les champs radiothérapeutiques afin d'inclure ces régions.

\section{TOMODENSITOMETRIE (SCANNER)}

Il arrive fréquemment que l'envahissement paramétrial (en particulier dans son tiers intérieur) et vaginal ne soit pas détecté. Les résultats faussement positifs sont également fréquents, car il est difficile de distinguer une invasion d'une inflammation, d'une réaction à une précédente irradiation ou d'une infection [19]. En revanche, la tomodensitométrie des cancers évolués du col (stades IIb a IV) semble capable d'améliorer les données cliniques, en définissant précisément le volume tumoral, en évaluant l'envahissement contigu dans les structures voisines et en permettant l'étude du foie et de l'appareil urinaire ${ }^{|6|}$. Une étude récente a ụindiqué une valeur prédictive positive de la tomodensitométrie de $60 \%$ pour l'envahissement vésical et une valeur prédictive négative de $100 \%$ [17. La tomodensitométrie peut permettre de visualiser directement les uretères, la région rétropéritonéale, les parois latérales pelviennes et les adénopathies. L'intestin peut être opacifié, et les structures vasculaires renforcées par un produit de contraste. Pour toutes ces raisons, la tomodensitométrie est utile dans le suivi du traitement.

Bien qu'elle ne puisse pas déceler l'envahissement de ganglions lymphatiques de taille normale, en particulier des ganglions pelviens, cette technique présente une bonne spécificité et sensibilité (environ 70 à $80 \%$ ) pour l'identification des ganglions lymphatiques para-aortiques anormaux ${ }^{12}$, 61. Idéalement, les ganglions positifs devraient être explorés par une biopsie par aspiration à l'aiguille fine ou une excision chirurgicale, car la tomodensitométrie est associée à 5 à $10 \%$ de résultats faux positifs [i].

\section{ECHOGRAPHIE}

Cette technique peut être employée pour l'exploration de l'appareil génito-urinaire et du système lymphatique. Elle est également utile pour différencier une obstruction lymphatique d'une thrombose veineuse profonde chez les patientes présentant un cedème de la jambe. L'utilisation actuelle de sondes transvaginales et/ou transrectales a considérablement amélioré la sensibilité de l'échographie lors de l'évaluation du volume tumoral et de son extension locale (paramètres, vessie). Cette approche est aussi adaptée au suivi du traitement. En outre, elle permet non seulement de détecter les hydronéphroses, mais également de les suivre durant la radiothérapie. L'échographie est toutefois moins sensible que la lymphographie et la tomodensitométrie pour la détection des ganglions lymphatiques. De plus, les résultats échographiques dépendent de l'opérateur et cette technique a peu de probabilité de fournir des données objectives et reproductibles pouvant être utilisés pour la classification du stade tumoral.

\section{IMAGERIE PAR RÉSONANCE MAGNETIQUE}

L'IRM semble constituer une technique prometteuse pour l'évaluation de l'envahissement paramétrial, avec une sensibilité de l'ordre de 85 à $92 \%$. Dans le cas de l'évaluation de l'espace vésicovaginal, sa sensibilité a été estimée entre 75 et $85 \%$ [6]. Elle permet de mesurer avec précision les tumeurs du col (profondeur de l'invasion stromale et volume tumoral) et de suivre le traitement, particulièrement aux stades avancés, mais également au stade $\mid \mathbf{b}{ }^{[15]}$. Même avec l'IRM haute résolution, une sensibilité de seulement $68 \%$ et une spécificité de seulement $78 \%$ ont été obtenues pour le diagnostic de l'envahissement des ganglions lymphatiques $[9]$.

\section{COMPARAISON DES EXAMENS OPTIONNELS}

Comparativement à l'IRM, la tomodensitométrie permet une bonne visualisation du thorax et de l'abdomen supérieur. Un autre avantage de la tomodensitométrie par rapport à l'IRM est la disponibilité d'un contraste intestinal et la possibilité de visualiser les métastases des voies urinaires et du foie. $A$ la différence de l'IRM, la tomodensitométrie et la biopsie par aspiration à l'aiguille fine sous guidage échographique permettent d'étudier la nature d'une lésion suspecte située dans le paramètre ou les ganglions lymphatiques et de confirmer une récidive tumorale. Cependant, l'IRM est plus adaptée que la tomodensitométrie pour distinguer le tissu cervical et utérin cancéreux du tissu normal. Par conséquent, l'IRM présente un plus grand intérêt dans l'établissement de schémas radiothérapeutiques optimaux.

Pour évaluer le volume tumoral et l'extension paramétriale et vésicovaginale, l'IRM est supérieure à la tomodensitométrie et à l'échographie, que ce soit en situation préthérapeutique ou de suivi k. 6l. Il est généralement admis que l'envahissement ganglionnaire est mieux évalué par une approche lymphographique suivie d'une tomodensitométrie. Cependant, les résultats d'une récente métaanalyse n'ont révélé aucune différence statistiquement significative entre la lymphographie, la tomodensitométrie et l'IRM, dans l'évaluation des métastases ganglionnaires [16!. En cas de suspicion d'une récidive, un examen tomodensitométrique doit être pratiqué. Enfin, employée en seconde ligne, l'IRM peut également aider à distinguer une fibrose d'une récidive de la maladie.

\section{LAPARASCOPIE}

Étant donné la discordance, allant de 30 à $70 \%$, entre la caractérisation clinique et les observations chirurgicales 118,201 , la laparoscopie peut améliorer la stadification du cancer du col. Elle peut permettre de diagnostiquer des métastases intrapéritonéales, annexielles ou hépatiques non suspectées ou passant inaperçues. Des biopsies et lavages intrapéritonéaux peuvent aider à faire la distinction entre inflammation et invasion. En outre, l'évaluation laparoscopique et le prélèvement dés ganglions lymphatiques pelviens et para-aortiques ont été décrits comme présentant une sensibilité d'environ $92 \%$ pour l'identification de la maladie ${ }^{[14]}$, ce qui est susceptible de modifier le traitement primaire proposé à la patiente.

\section{Classification chirurgicale et pathologique}

Certains facteurs (comme l'absence ou la présence et le taux de métastases lymphatiques, ou l'extension paramétriale subclinique) possédant une signification pronostique et ayant une incidence sur la sélection et la séquence des modalités thérapeutiques, ne sont pas pris en compte dans la classification clinique.

La classification chirurgicale des patientes présentant un cancer $\mathrm{du}$ col montre qu'une certaine proportion possède des métastases lymphatiques extrapelviennes situées hors des champs traditionnels $d$ 'irradiation. Une irradiation à champ élargi peut permettre de sauver certaines de ces patientes ${ }^{[13]}$. Cependant, la classification chirurgicale est susceptible de retarder la mise en route de la 
radiothérapie et d'augmenter les complications thérapeutiques (par exemple, thrombose veineuse profonde, embolie pulmonaire, occlusion intestinale). En outre, les nouvelles techniques d'imagerie, plus précises, peuvent éviter aux patientes atteintes d'un cancer du col le recours à une stadification chirurgicale, à laquelle est associée une certaine morbidité, voire une certaine mortalité.
Après l'intervention, les données pathologiques fournies par les échantillons prélevés peuvent servir de base à une évaluation très précise de l'étendue de la maladie : la classification pathologique. Ces résultats n'ont pas d'incidence sur la classification clinique, mais peuvent être utiles pour la prise en charge de la patiente et constituer des paramètres pronostiques intéressants.

\section{Références}

[1] Bandy LC, Clarke-Pearson DL, Stherman PM, Creasman WT. Computed tomography in evaluation of extrapelvic byphadenectomy in carcinoma of the cervix. Obstet Grinecol 1985;65:73-76

[2] Brenner DE, Whitley NO, Prempree T, Villasanta U. An evaluation of the computed tomographic scanner for the staging of carcinoma of the cervix. Concer 1982; 50 : 2323-2328

[3] Burghardt E, Ostö A, Fox H. The new FKGO definition of cervical cancer stage IA: a critique. Gmecol Oncol 1997; 65:1-5

[4] Cobby M, Browning I, Jones A, Whipp E, Coddard P. Ma gnetic resonance imaging, computed tomograpty and endosonography in the local staging of carcinoma of the cervix. A I Radio 1990;63:673-67\%

[5] Creasman WT. New gynecologic cancer staging. Gmecol Oncol $1995 ; 58: 157-158$

[6] Darbois Y, Buthiau D, Dargent D. Cancer du col uttérin. In : Buthiau D, Khayat Deds. Scanner et IRM en cancérologie. Berlin : Spinger-Verlag, 1995 : $279-290$

[7] Fuchs WA, Rosenberg CS. Lymphography in carcinoma of the uterine cervix. Acto Rodiol 1975; 16 : 353-361

[8] Hammond M, Herson I. Freedman RS, Hamberger AD, Wharton $\pi$, Wallace $S$ et al. The impact of tymph node status on survival in cervical carcinoma. Int / Rodiot Oncal siol Phy 1981; 7:1713-1718
[9] Hawighorst H, Schoenberg SO, Knapstein PC, Knopp MN, Schaeffer U, Essig M et al. Staging of invasive cervical carcinoma and of pelvic tymph nodes by high resolution MRI with a phased-array coil in comparison with pathological findings. / Comput Assist Tomogr 1998; 22 : 75-81

[10] Koelher PR, Wohl GT, Schaffer B. Lymphangiography. A survey of its current status. AlR Am / Roentgenol $1964 ; 91$ : $1216-1223$

[11] Leman MH Jr, Park RC, Baham ED, Chism SE, Petty WM, Patow WE. Pretreatment lymphanglography in carcinoma of the uterine cervix. Cynecol Oncol $1975 ; 3: 354-360$

[12] Five MS, Wallace S, Castro JR. The accuracy of bymphangiography in carcinoma of the uterine cervix. AAR Am I Roentgenol $1971 ; 111: 278-283$

[13] Podcraskj E, Stryker A, Kaminski P, Ndubisi B, Larson I Degeest $K$ et al. Extended-field radiation therapy for carcinoma of the cervix. Cancer $1990 ; 66: 251-258$

[14] Possover M, Krause N, Kuhne-Heid R, Schneider A Value of laparoscopic evaluation of para-aortic and pelvic bymph nodes for treatment of cervical cancer. Am J Obs Crmecol $1998 ; 178: 806-810$
[15] Rubens D, Thombury IR, Angel C, Stoler MH, Weiss SL, terner RM et al. Stage IB cervical carcinoma: comparison of clinical, MR, and pathologic staging. AVR Am / Roentgenol $1988 ; 150: 135-138$

[16] Scheidler ], Hricak H, Yu KK, Subak L, Segal MR. Radiological evaluation of bymph node metastases in patients with cervical cancer. A meta-analysis. JAMA 1997 ; 278 : 1096-1101

[17] Sundborg ML, Taylor RR, Mark I. Eg SA. Cystoscopy after computed tomography scan to ideritify bladder invasion in cervical cancer. Obstet Gymecol 1998;92:364-366

[18] Twiggs LB, Potish RA George R], Adcock LL Pretreatment extraperitoneal surgical staging in primary carcinoma of the cervix uteri. Surg Grnecol Obstet 1984;158:243-250

[19] Villasanta V, Whittley NO, Haney PL, Brenner D. Computed tomography in invasive carcinoma of the cervix: an appraisal. Obstet Gynecol 1983;62:218-224

[20] Wharton $\Gamma$, lones HW, Day TC $k r$, Rutledge FN, fletcher CH. Pre-irradiation celiotomy and extended field irradiation for imvasive carcinoma of the cervix. Obstet Gynecol $1977 ; 49: 333-338$ 